http://jmscr.igmpublication.org/home/

ISSN (e)-2347-176x ISSN (p) 2455-0450

crossref DOI: https://dx.doi.org/10.18535/jmscr/v7i8.63

\title{
Calcified Demoid Cyst Mimicking a Fibroid Mass in a 35 yr Old Woman with Secondary infertility
}

\author{
Authors \\ Achebe $\mathrm{CC}^{1}$, Adeniyi $\mathrm{AA}^{2^{*}}$, Adesiyun $\mathrm{OA}^{3}$, Aremu $\mathrm{IB}^{3}$, \\ Adeyemo OT ${ }^{2}$, Olabinri EO ${ }^{1}$, Ayankunle $\mathrm{MO}^{2}$ \\ ${ }^{1}$ Department of Radiology, Afe Babalola University/ Federal Teaching Hospital, Ido Ekiti, Nigeria \\ ${ }^{2}$ Department of Obstetrics and Gynaecology, Afe Babalola University/ Federal Teaching Hospital, Ido Ekiti, \\ Nigeria \\ ${ }^{3}$ Department of Radiology, University of Ilorin Teaching Hospital, Ilorin, Nigeria \\ *Corresponding Author \\ Adeniyi AA.
}

\section{Introduction}

Pelvic masses have become one of the common reasons why females present in the gynecological unit. Amongst these, uterine fibroid is one of the commonest ${ }^{1}$.

Degenerative changes, of which hyalinization is the commonest and calcification occurs in about $4 \%$ of fibroids. ${ }^{2}$ These calcifications are usually dense and amorphous, making them readily visible on plain abdominal radiographs. However, other related calcified masses can present in this way, especially when located in the pelvic cavity. A rare calcified dermoid cyst mass is one of such masses. ${ }^{3}$ Differentiating this from a fibroid on plain radiograph/ ultrasound could present a diagnostic challenge as in case presented below.

\section{Case Report}

A.L was a $35 y$ r old multiparous woman who presented to the Obstetrics and Gynecology unit of University of Ilorin teaching hospital with 8yr old history of secondary infertility. There was associated history of abdominal swelling, occasional abdominal pain and heavy menstrual flow. Examination revealed a palpable 16week size mass arising from the pelvis. An assessment of a uterine myoma was made. Ultrasound examination of the pelvis revealed multiple echogenic uterine masses. A super imposed huge cystic calcified mass measuring about $3 \mathrm{~cm} \times 3 \mathrm{~cm}$ with amorphous calcification was seen in the abdomen (fig 1). A plain abdominopelvic radiograph prior to hysterosalpingogram (HSG) showed a well defined calcified rounded mass within the pelvic cavity, while HSG revealed uterus with fundal bulge and non demonstration of both tubes most likely due to the masses (fig 2). A diagnosis of multiple myoma with blocked tubes and an associated calcified dermoid cyst to rule out pedunculated fibroid was made. This was further confirmed post surgery by the pathologist to be an associated dermoid cyst. Patient was discharged home after surgery post recovery to the clinic. 


\section{JMSCR Vol||07||Issue||08||Page 368-371||August}

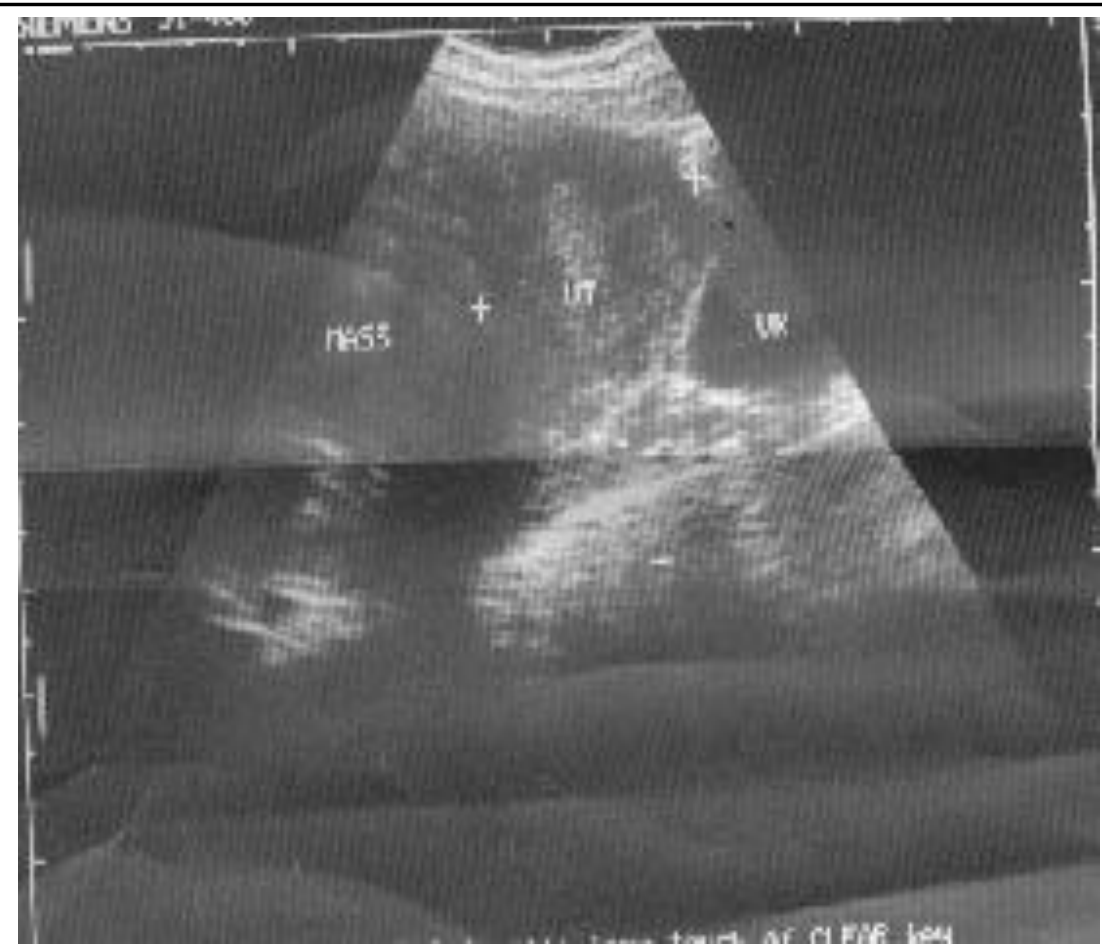

Figure 1 Ultrasonogram of the pelvis showing the huge adnexal mass with internal echoes and the slightly bulky uterus
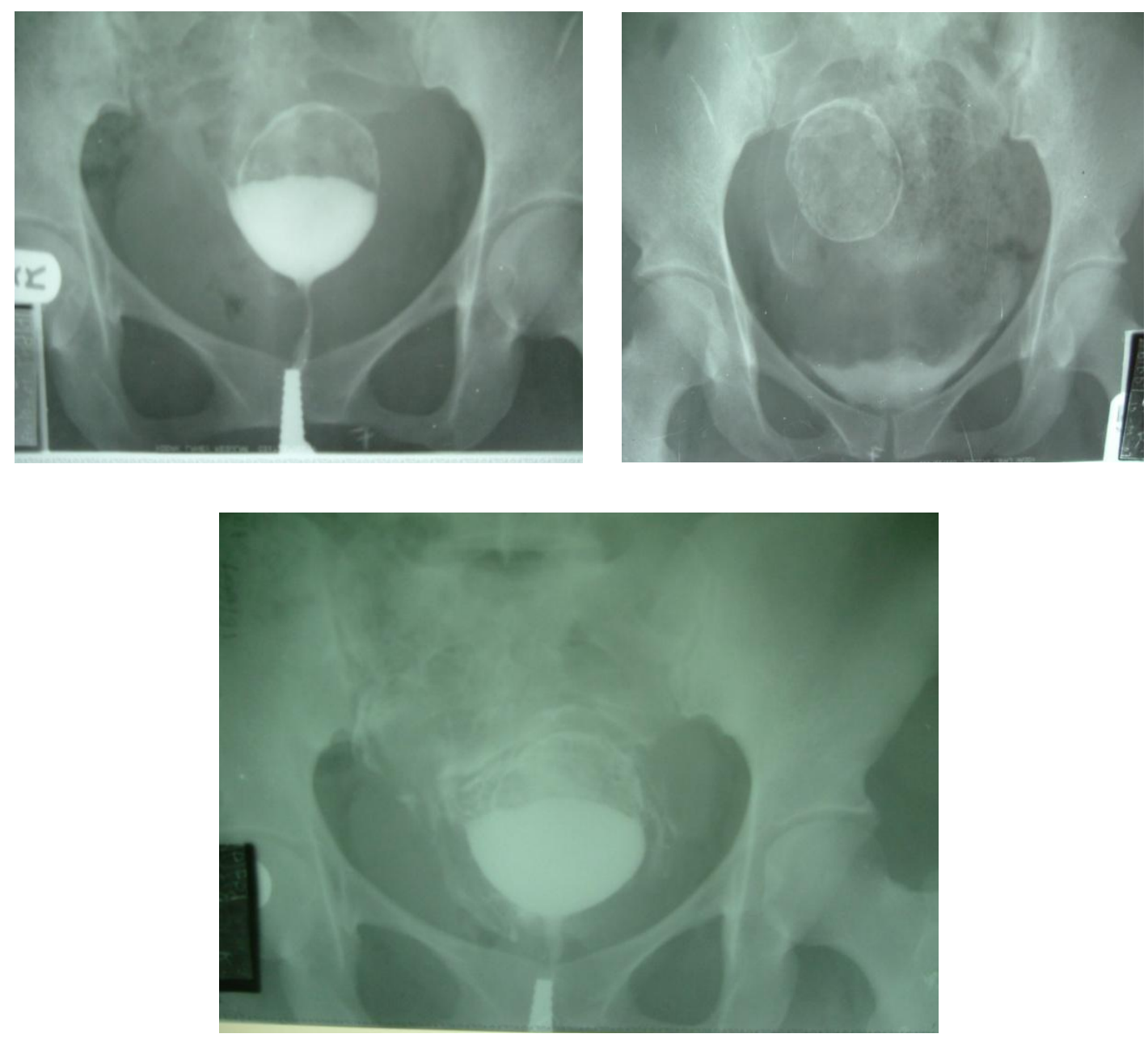
Figure 2 Hysterosalpingogram showing the calcified mass with internal (fluffy)_opacities_and a bulging fundus of the uterus and non demonstration of both fallopian tubes most likely from obstruction from the myomata.

\section{Discussion}

Leiomyomata are benign tumors of the muscle and connective tissue. They are hormone dependent, changing in size and echotexture during pregnancy and after menopause ${ }^{1,8}$. It is seen in about $40 \%$ of women beyond $35 \mathrm{yrs}$ of age $^{5}$. Aboyeji et $\mathrm{al}^{6}$ in Ilorin showed it to constitute $13.4 \%$ of all gynecological admissions and $78.4 \%$ are of ages between 30 and $44 \mathrm{yrs}$. Multiparity is a known predisposing factor ${ }^{1,} 6$. They could be: submucosal, intra mural, or subserous and rarely seen in the cervical region. Commonest presentations in order are those of menstrual disorder, infertility and lower abdominal swelling ${ }^{6}$. Others include habitual abortion and obstructed labour ${ }^{1}$. The latter were not seen in this patient. Complications in the subserous type when pedunculated include torsion, infarction and the classical "popcorn" calcification ${ }^{7}$. Degenerative changes account for most pathological and sonographic findings. Calcification may occur in about $25 \%$ of cases with high amplitude echo seen within mass or calcification around rim of tumor. ${ }^{1,7}$ as seen in patient. The combination of calcified mass with a peduncle can make it difficult to discern an ovarian mass dermoid cyst especially if calcified as seen in this patient. However, literature has it that calcified dermoid cyst are not as common as leiomyoma and are composed largely of ectodermal elements. ${ }^{8}$ They also tend to occur more in women of reproductive age group less than $35 \mathrm{yrs}$, but some cases in the prepubertal and elderly women have been reported ${ }^{9}$. They make up about $10-15 \%$ of ovarian tumors. Presenting symptoms include abdominal discomfort, pain and/or pressure as seen in this patient. Complications include torsion or rupture, though not seen in our patient ${ }^{9}$. Less than $1 \%$ are malignant. ${ }^{9}$ Dermoid cysts rarely calcify and when they do, they may show classification on plain radiograph. They show a distinct fibrous capsule, which may be recognized radiographically by virtue of contrasting effect of the greasy dermoid matter and surrounding peritoneal fat ${ }^{3}$. Plain radiograph of the abdomen/pelvis does not differentiate the duo of calcified fibroid or dermoid but may suggest the nature of the aforementioned form of popcorn calcification in fibroid. Ultrasonography of the abdomen may separate the two by virtue of location and attachment to the uterus. The composition of ectodermal structure like tooth which may cause increased echogenicity within the dermoid cyst may also be seen.

Contrast studies like HSG help to evaluate the status of uterus and possible complications associated with fibroid to assess the patency of fallopian tubes. Computed tomography (CT) apart from its advantages in assessing complications, helps to discern fat, calcification and ectodermal constituents specific to demoid cyst.

Magnetic resonance imaging (MRI) shows the fat delinated as hyperintense wall of the dermoid cyst and deficient calcification not seen in both cases when present ${ }^{1}$. However, complications are readily appreciated when present. The duo of MRI and CT were not done in patient due to financial constraint and non availability. In spite of these vast imaging modalities, the diagnosis of either dermoid cyst or a fibroid is hinged on pathological examination post surgery. Treatment modalities are majorly by surgery. However, new innovations have shown uterine artery embrolization in cases of myomas. Laparascopic removal of dermoid cyst is now being practiced in some countries ${ }^{9}$.

\section{Summary}

A case of a $35 \mathrm{yr}$ old multiparous woman who presented with history of infertility, abdominal mass and hemorrhage. Plain radiograph of abdomen, ultrasonography and HSG were highly suggestive of uterine myoma and dermoid cyst (calcified). She had surgical removal of the 


\section{JMSCR Vol||07||Issue||08||Page 368-371||August}

masses and histology confirmed uterine myomas with a calcified dermoid cyst. She was managed and discharged home for subsequent follow up.

\section{References}

1. Szu-Yung, Kuan-Gen Huang, Kee-My Yeow, Shang-Gwo Hong; Taiwanese J. of Obstetrics and Gyne.; March 2007; Vol 46(No 1): pg 85-87

2. Ueda H, Yogashik Kunishi et al; Unusual appearances of uterine leiomyomas: MR imaging findings and their histopathological backgrounds; Radiographics 1999; 19:5131-45

3. Pantoja, Sam Pedro C.A, Jiltivanich U; The radiographic wall signs of ovarian demoids; Rev. Inter. Radiol.; 1997 Jan; 2(1):33-5

4. Bu J.N, Bazot M, Ghossain M, Scot C, Hugol D, Bigot J.M et al; X ray computed tomographic aspect of demoid cyst of the ovary; Journal of radiology; 1989 Feb.; 70(2):103-9

5. David Sutton; A concise textbook of clinical imaging; 2nd edition; Mosby Yearbook (Inc), St Lous, Missouri; pg 635

6. Aboyeji Ijaya M.A; Uterine fibroid: A ten year clinical review in Ilorin, Nigeria; Niger. J. Med.; 2002 Jan-March; 11(1):169

7. Juhl-Paul and Juhl; Essentials of radiologic imaging, 7th edition; Lippincott Williams and Wilkins publishers; pg 28

8. Katie M. Williams, Charles J. Bain, Michael D. Kelly; Laparascopic resection of a torted ovarian demoid cyst; World journal of emergency surgery; 2007; 2:12

9. Berg C, Bendorff U, Diedrich K, Malik E; Laparascopic management of ovarian demoid cyst: A series of 83 cases; Arch. Gynecol. Obstet.; 2002; 266(3):126-9. 